\title{
Interpolation by a prime factor other than 2 in low-voltage low-power DAC
}

\author{
Pracný, Peter ; Jørgensen, Ivan Harald Holger; Chen, Liang; Bruun, Erik
}

Published in:

Proceedings of Norchip 2013

Link to article, DOI:

10.1109/NORCHIP.2013.6702024

10.1109/norchip.2013.6702024

Publication date:

2013

Link back to DTU Orbit

Citation (APA):

Pracný, P., Jørgensen, I. H. H., Chen, L., \& Bruun, E. (2013). Interpolation by a prime factor other than 2 in lowvoltage low-power DAC. In Proceedings of Norchip 2013 IEEE.

https://doi.org/10.1109/NORCHIP.2013.6702024, https://doi.org/10.1109/norchip.2013.6702024

\section{General rights}

Copyright and moral rights for the publications made accessible in the public portal are retained by the authors and/or other copyright owners and it is a condition of accessing publications that users recognise and abide by the legal requirements associated with these rights.

- Users may download and print one copy of any publication from the public portal for the purpose of private study or research.

- You may not further distribute the material or use it for any profit-making activity or commercial gain

- You may freely distribute the URL identifying the publication in the public portal 


\title{
Interpolation by a Prime Factor other than 2 in Low- Voltage Low-Power $\Sigma \Delta$ DAC
}

\author{
Peter Pracný, Ivan H. H. Jørgensen, Liang Chen and Erik Bruun \\ Department of Electrical Engineering \\ Technical University of Denmark \\ Kgs. Lyngby, Denmark \\ pp@elekto.dtu.dk
}

\begin{abstract}
This paper presents power optimization of a sigmadelta $(\Sigma \Delta)$ modulator based digital-to-analog converter (DAC) for hearing-aid audio back-end application. In a number of state-of-the-art publications the oversampling ratio (OSR) of the $\Sigma \Delta$ modulator is chosen as a factor of integer power of two. The reason given is the simplicity of the interpolation filter (IF) block. However, being able to choose OSR factors of integer powers of two only, might be restricting and not necessarily optimal. Therefore the $\Sigma \Delta$ modulator based DAC designs with multistage IF that include a stage performing oversampling by a factor of 3 are investigated. This new design freedom is used to lower the operating frequency of the whole DAC and save considerable amount of power. It is shown that the figure-ofmerit (FOM) of such designs can be lower than designs using oversampling by a factor of integer powers of two. The same optimization approach can be used for other low voltage low power portable audio applications (mobile phones, notebook computers etc.).
\end{abstract}

Keywords—sigma-delta modulator; interpolation filter; class D; hearing aid; low voltage, low power

\section{INTRODUCTION}

High audio quality, longer operation time and small device size are parameters demanded in hearing-aids today. Optimum balance between the design parameters in every part of a hearing-aid device is therefore of vital importance, making the power consumption one of the crucial parameters for the design. This is also the case of the audio signal processing path, which requires digital-to-analog conversion and power amplification at the back-end to drive the speaker (see Fig.1).
As part of the audio back-end a digital $\Sigma \Delta$ modulator with class D power amplifier (PA) is usually used in low-voltage low-power audio applications. Design specifications of such back-end intended for hearing-aid application are covered in Section II. The use of class D PA eliminates problems with device matching and reduced power efficiency experienced in case class AB PA is used [1,2]. The class D PA is usually implemented as an H-bridge (schematic in Fig.1 is simplified) and operates in switched mode with switching frequency $f_{s, P A}$. Compared to $[1,2]$ that use class $A B$ power stage, the class $D$ allows to perform all signal processing before the output filter in digital domain. Digital design provides the advantage of low-voltage low-power and cost effective implementation and scales down with integrated circuit (IC) technologies of today.

When using a multi-bit $\Sigma \Delta$ modulator with Q bits, digital pulse width modulation (DPWM) block that turns the $\Sigma \Delta$ signal into symmetrical 1 bit pulse width modulation, is needed. As can be seen in Fig. 1 the DPWM block requires the fastest clock in the back-end system and thus sets the system clock to $f_{s, D P W M}=2^{\mathrm{Q}}$. OSR . $f_{s}$, where $f_{s}$ is the input sampling frequency.

Due to the oversampling nature of the $\Sigma \Delta$ modulator an IF is needed prior to the modulator. In [3] it has been shown that with the class D PA being the main power consumer in the back-end and its switching frequency $f_{s, P A}=$ OSR $\cdot f_{s}$ depending on the OSR factor, decrease of the OSR results in considerable power savings. However, as will be shown in Section III of this work, the OSR decrease and the search for optimum design might be limited when the OSR has to be a

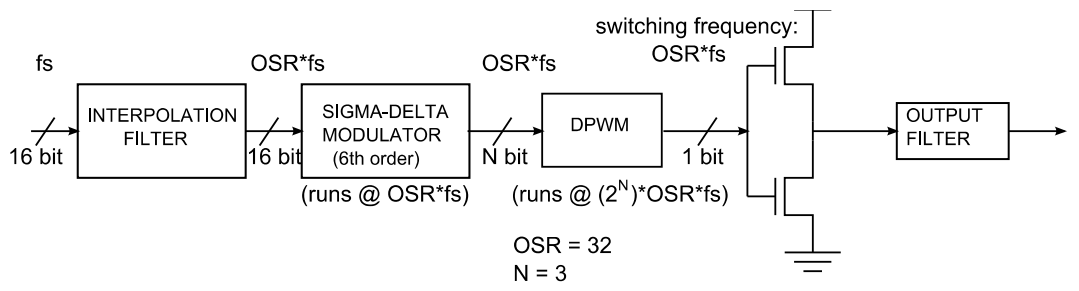

Figure 1. Simplified schematic of the back-end of audio signal processing chain: interpolation filter, $\Sigma \Delta$ modulator, class-D output-stage and output filter. 


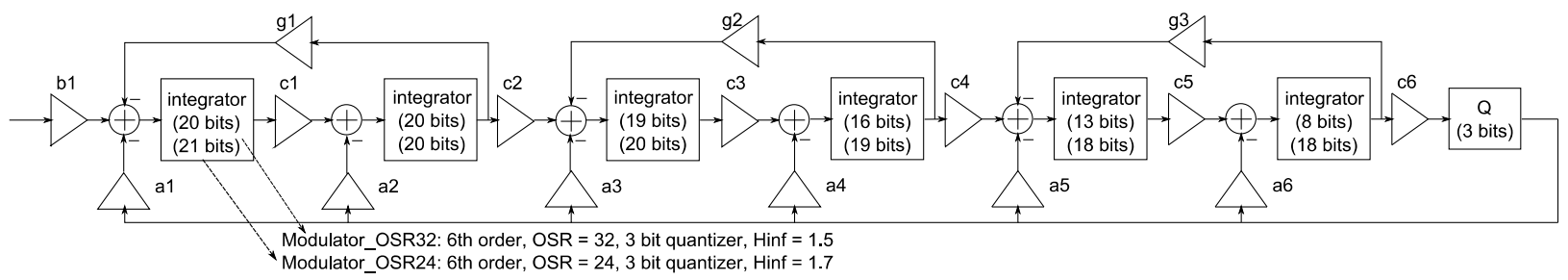

Figure 2. Simplified schematic of the $6^{\text {th }}$ order $\Sigma \Delta$ modulator.

factor of integer power of two as in [3 - 7]. To gain more design freedom a stage performing oversampling by a factor of 3 might be used as one of the stages of the IF. Such solution is discussed in Section IV along with simulation results and comparison with previous designs. Conclusion can be found in Section V.

\section{DESIGN AND FIGURE-OF-MERIT SPECIFICATIONS}

A thorough discussion on hearing-aid audio back-end system specification and the $\Sigma \Delta$ modulator is provided in [4]. Ideal 16 bit quantization of the system input signal is assumed. The input signal has band-width (BW) of $10 \mathrm{kHz}$. This results in signal-to-quantization-noise ratio $(\mathrm{SQNR})=98 \mathrm{~dB}$. The sampling frequency at the system input is $f s_{\text {in }}=22.05 \mathrm{kHz}$. The input signal of the back-end is then up-sampled using an IF and passed to the $\Sigma \Delta$ modulator. The IF in state-of-the-art designs [1 - 8] consists of multiple stages. Another requirement is the signal-to-noise-and-distortion ratio (SNDR) at the total output of the back-end of $90 \mathrm{~dB}$. We designed the IF and the $\Sigma \Delta$ modulator to keep the quality of the audio signal at SNDR $=98 \mathrm{~dB}$ so that a margin of $8 \mathrm{~dB}$ is left for the performance reduction introduced by the output stage. Maximum stable amplitude (MSA) at the input of the $\Sigma \Delta$ modulator is also a crucial parameter in hearing-aids, the lowest limit in this work is set to $-1.2 \mathrm{dBFS}$.

Note that the $\Sigma \Delta$ modulator in this work is fully digital and is treated as a digital filter. This allows judging the complexity and power savings of the $\Sigma \Delta$ modulator and the IF using the figure-of-merit:

$$
F O M=\Sigma_{i}\left(b_{i} . O S R_{i}\right)
$$

Where $i$ is the number of adders in the $\Sigma \Delta$ modulator block, $b_{i}$ is the number of bits used in individual adders and $O S R_{i}$ is the oversampling used for the individual adders. In the case of the $\Sigma \Delta$ modulator block $O S R_{i}$ is the same for all the adders. Since most of power consumption in the IF and the $\Sigma \Delta$ modulator is caused by the adders, the FOM is approximately proportional to power consumption. There are more precise figures of merit for $\Sigma \Delta$ modulators used in other works [8]. However, these figures of merit can be used only after the design has been completed and possibly measured. The advantage of the figure of merit of Eq. 1 is that it allows comparison of different designs early in the design process allowing critical system design decisions.

\section{INTERPOLATION BY A FACTOR OF INTEGER POWER OF TWO}

Fig.1 shows a $\Sigma \Delta$ modulator based DAC that will be optimized with respect to power. The system level parameters of the $\Sigma \Delta$ modulator used in this DAC (see Fig. 2, Modulator_OSR32) [3] are $6^{\text {th }}$ order, OSR $=32,3$ bit quantizer. Maximum noise transfer function (NTF) gain $H_{\text {inf }}$ $=1.5$ is used as advised in [8]. The coefficients of this $\Sigma \Delta$ modulator can be seen in Tab. I. Fig.3(a) shows the IF (IF_OSR32) used for the $\Sigma \Delta$ modulator of Fig. 2, Modulator_OSR32. The IF consists of 4 stages and performs oversampling by 32 in total. The first two stages are designed as IIR filters as a parallel connection of two all-pass filter cells (see Fig. 4 and Fig. 5). The coefficients used in these filters can be found in Tab. II and Tab. III. The third stage is designed as a $3^{\text {rd }}$ order cascaded-integrator-comb (CIC) filter and the fourth stage as a second order CIC filter [8].

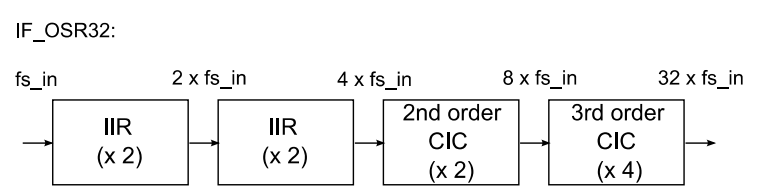

IF OSR24a:
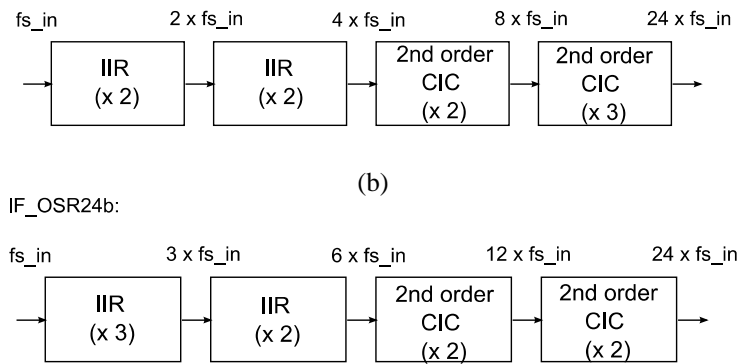

IF_OSR24c:

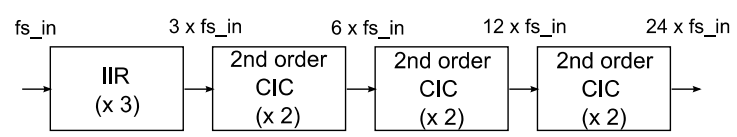

(d)

Figure 3. Multistage interpolation filters compared in this work. 
TABLE I. COEFFICIENTS OF THE $\Sigma \Delta$ MODULATOR OF FIG.2, MODULATOR_OSR32

\begin{tabular}{|c|c|c|c|}
\hline Coefficient & Value & Shift / Add & Adders \\
\hline $\mathrm{a}_{1}$ & $1 / 16$ & $2^{-4}$ & 0 \\
\hline $\mathrm{a}_{2}$ & 0.1542 & $2^{-3}$ & 0 \\
\hline $\mathrm{a}_{3}$ & 0.1705 & $2^{-3}+2^{-5}$ & 1 \\
\hline $\mathrm{a}_{4}$ & 0.2532 & $2^{-2}$ & 0 \\
\hline $\mathrm{a}_{5}$ & 0.5544 & $2^{-1}+2^{-5}$ & 1 \\
\hline $\mathrm{a}_{6}$ & 0.6353 & $2^{-1}+2^{-3}$ & 1 \\
\hline $\mathrm{b}_{1}$ & $1 / 16$ & $2^{-4}$ & 0 \\
\hline $\mathrm{c}_{1}$ & $1 / 8$ & $2^{-3}$ & 0 \\
\hline $\mathrm{c}_{2}$ & $1 / 8$ & $2^{-3}$ & 0 \\
\hline $\mathrm{c}_{3}$ & $1 / 4$ & $2^{-2}$ & 0 \\
\hline $\mathrm{c}_{4}$ & $1 / 2$ & $2^{-1}$ & 0 \\
\hline $\mathrm{C}_{5}$ & $1 / 2$ & $2^{-1}$ & 0 \\
\hline $\mathrm{C}_{6}$ & 0.8791 & $2^{0}-2^{-3}$ & 1 \\
\hline $\mathrm{g}_{1}$ & 0.0044 & $2^{-8}$ & 0 \\
\hline $\mathrm{g}_{2}$ & 0.0168 & $2^{-6}$ & 0 \\
\hline $\mathrm{g}_{3}$ & 0.0167 & $2^{-6}$ & 0 \\
\hline
\end{tabular}

TABLE II. COEFFICIENTS OF THE FIST STAGE OF IF_OSR32 (FIG. 3(A))

\begin{tabular}{|c|c|c|c|}
\hline Coefficient & Value & Shift / Add & Adders \\
\hline $\mathbf{a}_{\mathbf{1}, \mathbf{3}}$ & 0.9375 & $2^{-1}-2^{-4}$ & 1 \\
\hline $\mathbf{a}_{\mathbf{0}, \mathbf{3}}$ & 0.8047 & $2^{-1}+2^{-2}+2^{-4}-2^{-7}$ & 3 \\
\hline $\mathbf{a}_{\mathbf{1}, \mathbf{2}}$ & 0.6406 & $2^{-1}+2^{-3}+2^{-6}$ & 2 \\
\hline $\mathbf{a}_{\mathbf{0}, \mathbf{2}}$ & 0.4453 & $2^{-1}-2^{-4}+2^{-7}$ & 2 \\
\hline $\mathbf{a}_{\mathbf{1}, \mathbf{1}}$ & 0.2422 & $2^{-2}-2^{-7}$ & 1 \\
\hline $\mathbf{a}_{\mathbf{0}, \mathbf{1}}$ & 0.0703 & $2^{-4}+2^{-7}$ & 1 \\
\hline
\end{tabular}

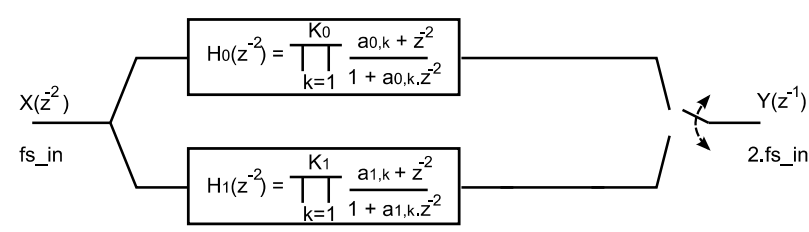

Figure 4. IIR filter using a parallel connection of two all-pass cells. Used as the first stage of IF_OSR32 (Fig. 3(a)).

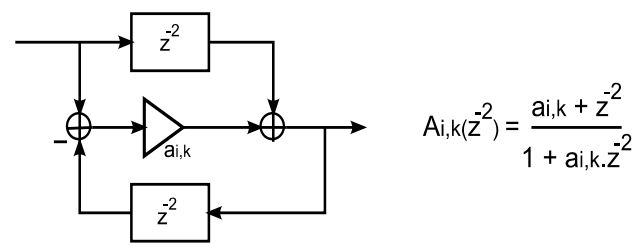

Figure 5. Second-order all-pass filter cell and its transfer function.

TABLE III. COEFFICIENTS OF THE SECOND STAGE OF IF_OSR32 (FIG. 3(A))

\begin{tabular}{|c|c|c|c|}
\hline Coefficient & Value & Shift / Add & Adders \\
\hline $\mathbf{a}_{\mathbf{1} \mathbf{1}}$ & 0.9375 & $2^{-1}+2^{-4}+2^{-6}$ & 2 \\
\hline $\mathbf{a}_{\mathbf{0}, \mathbf{1}}$ & 0.1348 & $2^{-3}+2^{-7}+2^{-9}$ & 2 \\
\hline
\end{tabular}

A model of this design using fixed-point arithmetic has been built and simulated in Matlab [3]. This model is transferable to VHDL. FFT spectrum of the $\Sigma \Delta$ modulator output signal is in Fig. 6, the transfer functions of the IF and the $\Sigma \Delta$ modulator are in Fig. 7. The FOM of the $\Sigma \Delta$ modulator and individual stages of the IF was calculated according to Eq. 1 and can be seen in Tab. IV.

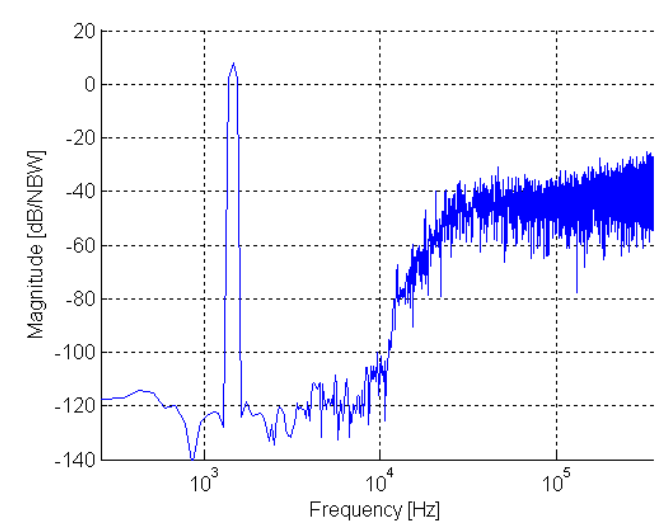

Figure 6. Output signal FFT spectrum of the $\Sigma \Delta$ modulator design Modulator_OSR32 (Fig.2). NBW = 1.8311e-04.

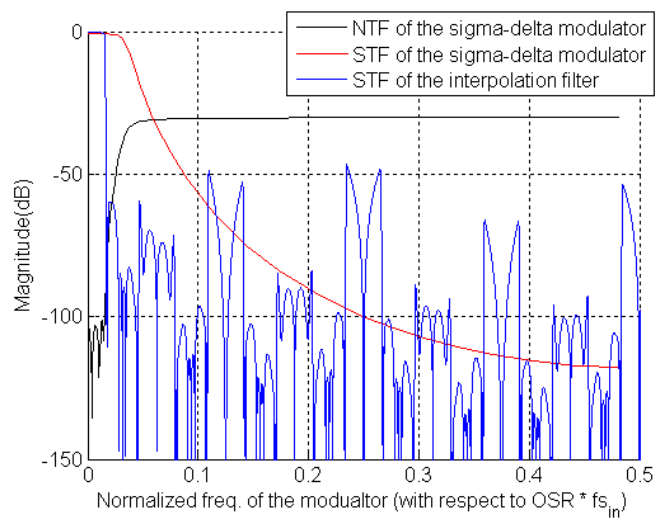

Figure 7. Transfer functiond of the $\Sigma \Delta$ modulator and the interpolation filter of Tab IV.

TABLE IV. FOM OF THE INDIVIDUAL BLOCKS OF IF AND OF THE $\Sigma \Delta$ MODULATOR

\begin{tabular}{|c|c|c|c|c|}
\hline IF design & IF_OSR32 & IF_OSR24a & IF_OSR24b & IF_OSR24c \\
\hline IF stage 1 & 9.5 & 9.5 & 19.9 & 19.9 \\
\hline IF stage 2 & 7.2 & 7.2 & 10.8 & 3.4 \\
\hline IF stage 3 & 8.6 & 4.5 & 6.7 & 6.7 \\
\hline IF stage 4 & 25.5 & 53 & 13.5 & 13.5 \\
\hline IF total & 51 & 74 & 51 & 43.5 \\
\hline$\Sigma \Delta$ modulator & 192 & 180 & 180 & 180 \\
\hline IF $+\Sigma \Delta$ & 243 & 254 & 231 & 223.5 \\
\hline
\end{tabular}

The goal is to optimize the DAC with respect to power compared to the design of [3] by reducing the OSR of the $\Sigma \Delta$ modulator. If the OSR is restricted to be a factor of integer power of two the only option is to reduce the OSR from 32 down to 16 . Such optimization would reduce the switching 
frequency of the Class D PA by $50 \%$ and thus save $50 \%$ of power compared to the design of [3]. Moreover the power consumption of the DPWM block would also be reduced by $50 \%$ as its operating frequency $f s_{D P W M}=2^{\mathrm{Q}}$. OSR $\cdot f_{s}$ depends directly on OSR. Power consumption would also be saved in the IF because the last stage that increases the frequency from $16 . f_{s \_ \text {in }}$ to $32 . f_{s \_ \text {in }}$ would not be needed. Tab IV shows that this stage has the highest FOM of all stages and thus consumes the largest amount of power in the IF. The only block of the DAC that remains to be investigated to see whether or not this optimization approach is reasonable is the $\Sigma \Delta$ modulator.

For this reason a plot of achievable peak SQNR for $\Sigma \Delta$ modulator with 3 bit quantizer as a function of OSR for orders $1-8$ is shown in Fig. 8 .

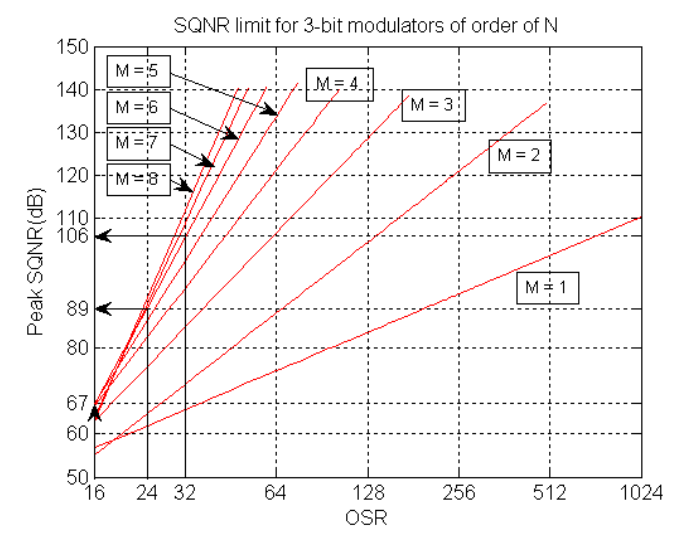

Figure 8. peak SQNR of the 3 bit $\Sigma \Delta$ modulator output signal as a function of OSR for modulator orders 1-8.

It can be seen that the design of Fig.2, Modulator_OSR32 achieves $106 \mathrm{~dB}$ peak SQNR. Since only $98 \mathrm{~dB}$ SQNR is needed at the output of the $\Sigma \Delta$ modulator according to the specification in Section II this leaves $106-98=8 \mathrm{~dB}$ for

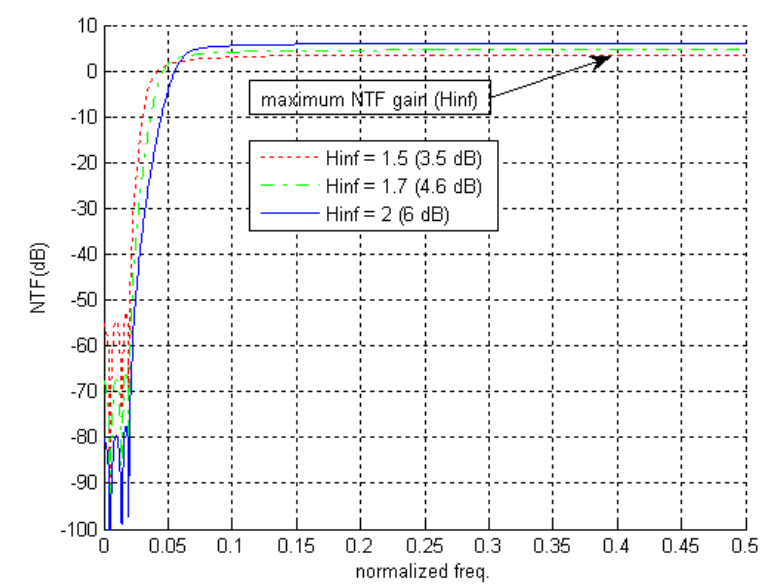

Figure 9. Raising the cutoff frequency of the $\Sigma \Delta$ modulator loop filter by increasing the maximum NTF gain of the $\Sigma \Delta$ modulator.

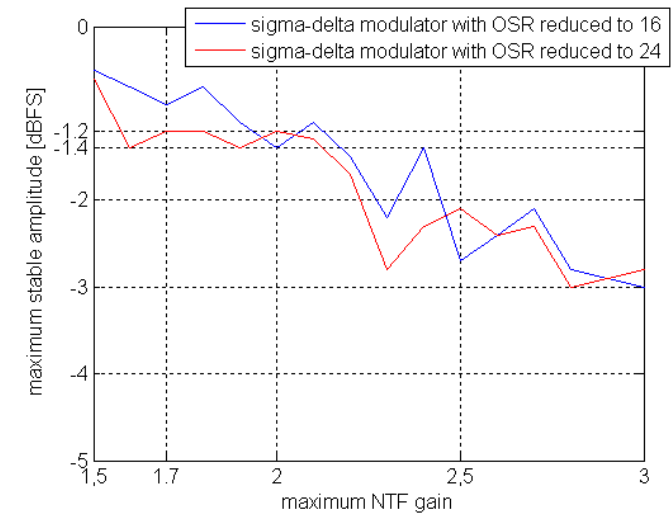

Figure 10. Maximum stable amplitude at $\Sigma \Delta$ modulator input as a function of max. NTF gain.

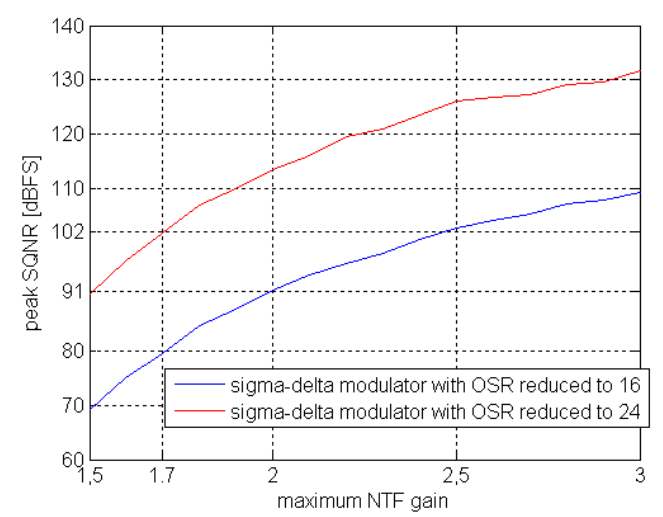

Figure 11. peak SQNR of the $\Sigma \Delta$ modulator output signal as a function of max. NTF gain.

performance reduction by coefficient quantization [3]. If the OSR is reduced from 32 to 16 the achievable peak SQNR drops from $106 \mathrm{~dB}$ to $67 \mathrm{~dB}$, not fulfilling the specification. In order to improve the SQNR the cutoff frequency of the $\Sigma \Delta$ modulator loop filter must be raised. This can be done by increasing the maximum NTF gain $H_{\text {inf }}$ of the $\Sigma \Delta$ modulator (see Fig. 9). However, at the same time, increase of the maximum NTF gain of the $\Sigma \Delta$ modulator reduces the MSA. The blue plot of Fig. 10 shows that at maximum NTF gain = 2 the MSA drops below the specification of $-1.2 \mathrm{dBFS}$ but the peak SQNR in the blue plot of Fig. 11 reaches only $91 \mathrm{~dB}$, still below the specification. This shows that the reduction of the OSR from 32 to 16 brings the design out of specification and is not acceptable. Therefore if the DAC has to be optimized with respect to power by lowering the OSR factor, the OSR has to be lower than 32 but higher than 16 e.g. a factor that is not an integer power of two. This solution will be discussed in the next section. 


\section{INTRODUCING INTERPOLATION BY A FACTOR OF 3}

By introducing a stage performing interpolation by a factor of 3 the OSR can be reduced from 32 down to 24. In such case the $\Sigma \Delta$ modulator is $6^{\text {th }}$ order with 3 bit quantizer, OSR $=24$ and maximum NTF gain $H_{\text {inf }}=1.5$. However Fig.5 shows again that if $H_{\text {inf }}=1.5$ is used as advised in [8] the modulator will reach only $89 \mathrm{~dB}$ peak SQNR, which is below the specification of Section II. This time increasing the maximum NTF gain helps to reach above the required $98 \mathrm{~dB}$ SQNR before the MSA drops below -1.2 dBFS (see Fig.10 red plot and Fig.11 red plot). $H_{\text {inf }}=1.7$ is used for the optimized $\Sigma \Delta$ modulator. Simplified schematic of the $\Sigma \Delta$ modulator is in Fig. 2, Modulator_OSR24. A model of this design using fixedpoint arithmetic has been built and simulated in Matlab. The model is transferable to VHDL. FFT spectrum of the $\Sigma \Delta$ modulator output signal is in Fig. 12. The coefficients of this optimized $\Sigma \Delta$ modulator can be found in Tab. V.

TABLE V. COEFFICIENTS OF MODULATOR_OSR24 (FIG.2)

\begin{tabular}{|c|c|c|c|}
\hline Coefficient & Value & Shift / Add & Adders \\
\hline $\mathrm{a}_{1}$ & $1 / 16$ & $2^{-4}$ & 0 \\
\hline $\mathrm{a}_{2}$ & 0.1172 & $2^{-3}-2^{-7}$ & 1 \\
\hline $\mathrm{a}_{3}$ & 0.0977 & $2^{-4}+2^{-5}+2^{-8}$ & 2 \\
\hline $\mathrm{a}_{4}$ & 0.1094 & $2^{-3}-2^{-6}$ & 1 \\
\hline $\mathrm{a}_{5}$ & 0.1875 & $2^{-3}+2^{-4}$ & 1 \\
\hline $\mathrm{a}_{6}$ & 0.1563 & $2^{-3}+2^{-5}$ & 1 \\
\hline $\mathrm{b}_{1}$ & $1 / 16$ & $2^{-4}$ & 0 \\
\hline $\mathrm{c}_{1}$ & $1 / 8$ & $2^{-3}$ & 0 \\
\hline $\mathrm{c}_{2}$ & $1 / 8$ & $2^{-3}$ & 0 \\
\hline $\mathrm{c}_{3}$ & $1 / 4$ & $2^{-2}$ & 0 \\
\hline $\mathrm{c}_{4}$ & $1 / 2$ & $2^{-1}$ & 0 \\
\hline $\mathrm{c}_{5}$ & $1 / 2$ & $2^{-1}$ & 0 \\
\hline $\mathrm{c}_{6}$ & 3.8750 & $2^{2}-2^{-3}$ & 1 \\
\hline $\mathrm{g}_{1}$ & 0.0078 & $2^{-7}$ & 0 \\
\hline $\mathrm{g}_{2}$ & 0.0313 & $2^{-5}$ & 0 \\
\hline $\mathrm{g}_{3}$ & 0.0293 & $2^{-5}-2^{-9}$ & 1 \\
\hline
\end{tabular}

The IF stage performing interpolation by 3 can be either the last CIC filter (see IF_OSR24a, Fig. 3(b)) or the first IIR filter (see IF_OSR24b, Fig.3(c)). In the case of IF_OSR24a the first two stages are reused from IF_OSR32. The third and fourth stage is second order CIC filter. The FOM of the $\Sigma \Delta$ modulator and individual stages of the IF was again calculated according to Eq. 1 and can be seen in Tab. IV.

Tab. IV shows that the IF_OSR24a and the $\Sigma \Delta$ modulator Modulator_OSR24 have worse FOM than in the case of OSR $=32$, but still by lowering the OSR from 32 to 24 the power consumption of the DPWM block and the main power consumer - the Class D PA is lowered by $25 \%$, yielding an overall power reduction. Tab. IV also shows that the largest contribution to FOM of the IF_OSR24a comes from the last stage. The reason for this is that it performs oversampling by a factor of 3 which makes it more complex compared to the situation of IF_OSR32. To improve the FOM further the stage performing interpolation by a factor of 3 can be the first stage IIR filter instead of the last stage CIC filter (see IF_OSR24b, Fig. 3(c)).

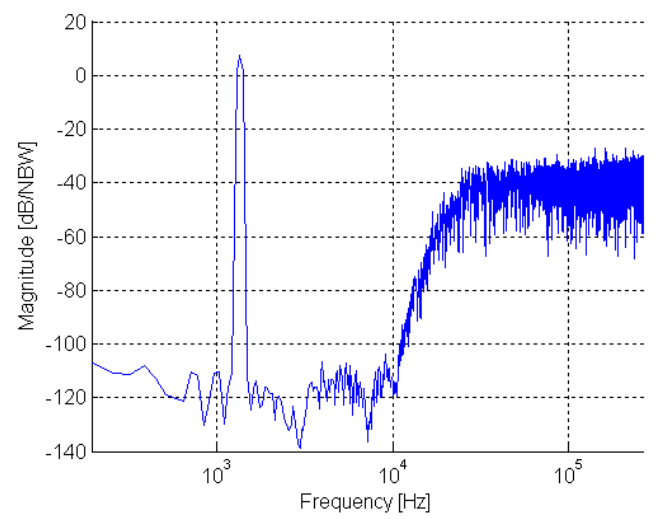

Figure 12. Output signal FFT spectrum of the $\Sigma \Delta$ modulator design Modulator_OSR24 (Fig.2). NBW = 1.8311e-04.

TABLE VI. COEFFICIENTS OF THE FIST STAGE OF IF_OSR24B (FIG. 3(C))

\begin{tabular}{|c|c|c|c|}
\hline Coefficient & Value & Shift / Add & Adders \\
\hline $\mathbf{a}_{2,3}$ & 0.9587 & $2^{0}-2^{-5}-2^{-7}-2^{-9}-2^{-12}$ & 4 \\
\hline $\mathbf{a}_{\mathbf{1}, \mathbf{3}}$ & 0.8892 & $2^{0}-2^{-3}+2^{-6}-2^{-9}+2^{-11}$ & 4 \\
\hline $\mathbf{a}_{\mathbf{0}, \mathbf{3}}$ & 0.7773 & $2^{0}-2^{-2}+2^{-5}-2^{-8}$ & 3 \\
\hline $\mathbf{a}_{2, \mathbf{2}}$ & 0.6592 & $2^{-1}+2^{-3}+2^{-9}+2^{-7}+2^{-10}$ & 4 \\
\hline $\mathbf{a}_{\mathbf{1}, \mathbf{2}}$ & 0.5151 & $2^{-1}+2^{-6}-2^{-11}$ & 2 \\
\hline $\mathbf{a}_{\mathbf{0}, \mathbf{2}}$ & 0.3652 & $2^{-1}-2^{-3}-2^{-7}-2^{-9}$ & 3 \\
\hline $\mathbf{a}_{2, \mathbf{1}}$ & 0.2207 & $2^{-2}-2^{-5}+2^{-9}$ & 2 \\
\hline $\mathbf{a}_{\mathbf{1}, \mathbf{1}}$ & 0.1016 & $2^{-4}+2^{-5}+2^{-7}$ & 2 \\
\hline $\mathbf{a}_{\mathbf{0}, \mathbf{1}}$ & 0.0303 & $2^{-5}-2^{-10}$ & 1 \\
\hline
\end{tabular}

In such case the first stage (IIR filter) is designed as a parallel connection of three second-order all-pass filter cells (see Fig. 12). The second-order all-pass filter cell used is in Fig.5. Coefficients of the first stage IIR filter can be found in Tab.VI. Again the FOM of the $\Sigma \Delta$ modulator and individual stages of the IF was calculated according to Eq. 1 and can be seen in Tab. IV.

Moreover a second order CIC filter can be used instead of the IIR filter in second stage (see IF_OSR24c, Fig. 3(d)). In this case the first stage of IF_OSR24b is reused and the remaining stages are second order CIC filters. The FOM of the $\Sigma \Delta$ modulator and individual stages of the IF was again calculated according to Eq. 1 and can be seen in Tab. IV.

For comparison a summary of the designs used in this work is

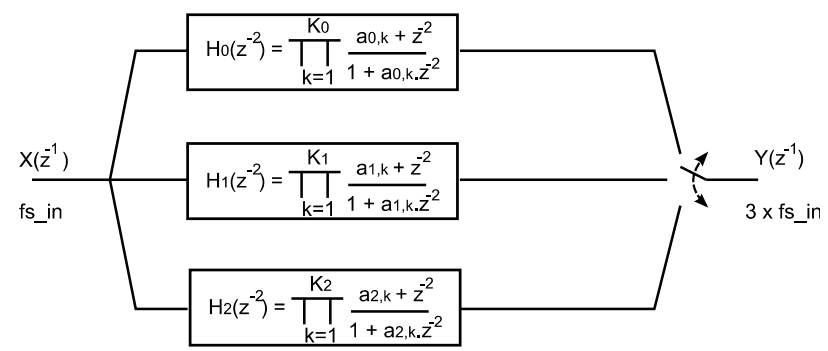

Figure 12. IIR filter using a parallel connection of three all-pass cells. Used as the first stage of IF_OSR24b (Fig. 3(c)). 
provided in Tab.VII. The transfer functions of the optimized Modulator_OSR24 and the three IFs of IF_OSR24b/c/d are in Fig.12. The peak-SQNR and the MSA of the Modulator_OSR24 (Fig.2), in the Matlab model using fixedpoint arithmetic was the same, no matter which one of the three IFs was used. The peak of the IF transfer function reaching above the $\Sigma \Delta$ modulator NTF in the case of IF_OSR24b and IF_OSR24c is shown not to be a problem in the case of interpolation. However, in the case of decimation it could cause problems with down-folding of noise. In the caae of interpolation, the difference is only in FOM of the IFs and their pass-band ripple, favoring the IF_OSR24d despite of the larger pass-band ripple, as $0.6 \mathrm{~dB}$ is within the specification of a hearing-aid.

TABLE VII. COMPARISON OF THE $\Sigma \Delta$ MODULATOR AND IF DESGNS

\begin{tabular}{|c|c|c|c|c|}
\hline Design & IF_OSR32 & IF_OSR24a & IF_OSR24b & IF_OSR24c \\
\hline $\begin{array}{c}\text { FOM (IF + } \Sigma \Delta \\
\text { modulator) }\end{array}$ & 243 & 254 & 231 & 223.5 \\
\hline $\begin{array}{c}\text { DPWM } \\
\text { frequency }\end{array}$ & $5.65 \mathrm{MHz}$ & $4.23 \mathrm{MHz}$ & $4.23 \mathrm{MHz}$ & $4.23 \mathrm{MHz}$ \\
\hline $\begin{array}{c}\text { Class D PA } \\
\text { switching } \\
\text { frequency }\end{array}$ & 705 & 529 & $\begin{array}{c}529 \\
\mathrm{kHz}\end{array}$ & $\begin{array}{c}529 \\
\mathrm{kHz}\end{array}$ \\
\hline $\begin{array}{c}\text { IF pass-band } \\
\text { ripple }\end{array}$ & $0.5 \mathrm{~dB}$ & $0.5 \mathrm{~dB}$ & $0.5 \mathrm{~dB}$ & $0.6 \mathrm{~dB}$ \\
\hline
\end{tabular}

\section{CONCLUSION}

This work shows that the optimized design with OSR factor other than integer power of two (OSR $=24$ ) has $25 \%$ operating frequency reduction in the DPWM block and the class D PA compared to the original design. Thus these blocks consume $25 \%$ less power while the audio quality has been kept within specifications. Based on the FOM results, power is saved if the stage performing interpolation by a prime factor other than 2 is implemented as the first stage IIR filter rather than the last stage CIC filter. The combined power consumption of the IF and the $\Sigma \Delta$ modulator was reduced by $8 \%$. In total considerable power savings were achieved. Therefore OSR factors other than integer powers of two should be considered when optimizing a $\Sigma \Delta$ modulator based DAC for low-voltage low-power portable audio applications.

\section{REFERENCES}

[1] K. Lee, Q. Meng, T. Sugimoto, K. Hamashita, K. Takasuka, S. Takeuchi, U. Moon, G. C. Temes, “A 0.8 V, $2.6 \mathrm{~mW}, 88 \mathrm{~dB}$ DualChannel Audio Delta-Sigma D/A Converter With Headphone Driver" IEEE Journal of Solid-State Circuits, Vol. 44, No. 3, Mar. 2009.

[2] K. Wong, K. Lei, S. U, R. P. Martins, “A 1-V 90dB DR Audio Stereo DAC with embedding Headphone Driver" IEEE Asia Pacific Conference Circuits snd Systems (APCCAS), Dec. 2008.

[3] P. Pracný, I. H. H. Jørgensen, E. Bruun, “System-Level Optimization of a DAC for Hearing-Aid Audio Class D Output Stage” 4th Doctoral Conference on Computing, Electrical and Industrial Systems (DoCEIS), Feb. 2013.

[4] P. Pracný, E. Bruun, " $\Sigma \Delta$ Modulator System-Level Considerations for Hearing-Aid Audio Class-D Output Stage Application,” Proc. 2012 8th Conf. on Ph.D. Research in Microelectronics and Electronics (PRIME), pp. 103-106, Aachen, Jun. 2012.

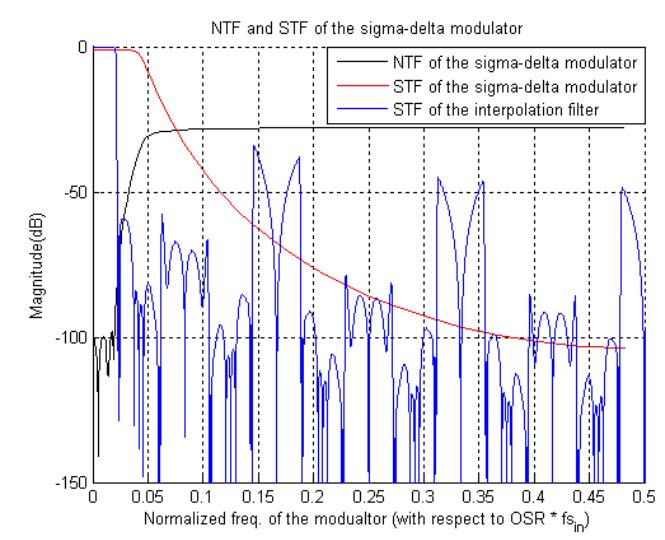

(a)

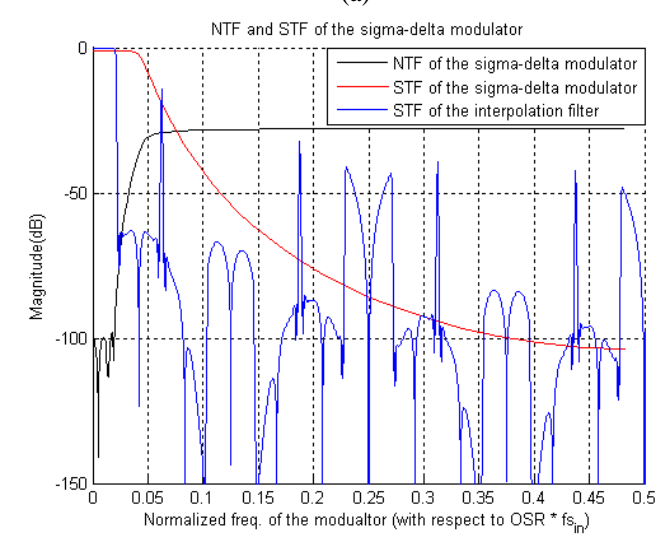

(b)

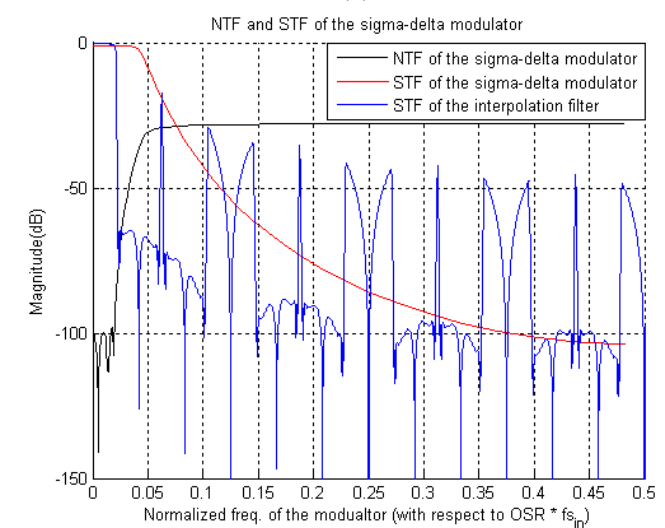

(c)

Figure 12. Transfer functions of the $\Sigma \Delta$ modulator Modulator_OSR24 (Fig.2) and the interpolation filter of (a) IF_OSR24a, (b) IF_OSR24b and (c) IF_OSR24c.

[5] T. Forzley, R. Mason, “A Scalable Class D Audio Amplifier for Low Power Applications,” Proc. AES 37th International Conference, Aug. 2009.

[6] X. Yao, L. Liu, D. Li, L. Chen, Z. Wang “A 90dB DR Audio DeltaSigma DAC with Headphone Driver for Hearing Aid” 3rd International Congress on Image and Signal Processing (CISP), 2010.

[7] S. Kim, N. Cho, S. Song, H. Yoo, “A 0.9 V 0.96 uW Fully Operational Hearing Aid Chip” IEEE Journal of Solid-State Circuits, Vol. 42, No. 11, Feb. 2007.

[8] R. Schreier, G. C. Temes, "Understanding Delta-Sigma Data Converters,” Wiley-IEEE Press, 2005. 